\title{
Al-Khayyâm mathématicien. Paris, Librairie scientifique et technique Albert Blanchard (Collection science dans l'histoire), 1999, x + 428 p.
}

\section{Hossein Masoumi-Hamedani}

\section{(2) OpenEdition}

1 Journals

Édition électronique

URL : http://journals.openedition.org/abstractairanica/36949

DOI : 10.4000/abstractairanica.36949

ISSN : 1961-960X

Éditeur :

CNRS (UMR 7528 Mondes iraniens et indiens), Éditions de l'IFRI

\section{Édition imprimée}

Date de publication : 15 mai 2001

ISSN : 0240-8910

Référence électronique

Hossein Masoumi-Hamedani, "Al-Khayyâm mathématicien. Paris, Librairie scientifique et technique Albert Blanchard (Collection science dans I'histoire), 1999, x + 428 p. », Abstracta Iranica [En ligne], Volume 22 | 2001, document 436, mis en ligne le 17 février 2010, consulté le 09 octobre 2020. URL http://journals.openedition.org/abstractairanica/36949; DOI : https://doi.org/10.4000/ abstractairanica.36949

Ce document a été généré automatiquement le 9 octobre 2020.

Tous droits réservés 


\title{
Al-Khayyâm mathématicien. Paris, Librairie scientifique et technique Albert Blanchard (Collection science dans l'histoire), 1999, $\mathrm{x}+428 \mathrm{p}$.
}

\author{
Hossein Masoumi-Hamedani
}

1 Ce livre présente une édition critique, à partir de tous les manuscrits connus, de presque tout ce qui nous est parvenu de l'œuvre mathématique de Hुayyām: traité d'algèbre (pp. 116-237), traité sur «la division d'un quart de cercle» (pp. 238-67), et «commentaire sur les difficultés de certains postulats de l'ouvrage d'Euclide » (pp. 305-83). Chaque traité est accompagné d'une traduction méticuleuse en français et précédé d'un commentaire mathématique dans lequel l'auteur (Rashed, pp.31-113, pour les deux premiers traités et Vahabzadeh, pp. 281-99, pour le troisième) explique, dans un langage accessible au lecteur moderne, ce que dit Hुayyām dans le langage mathématique de son temps. Les "notes complémentaires » (pp. 385-90), traitent de certaines questions historiques, linguistiques, et philosophiques qui déborderaient le cadre de ces commentaires, et un glossaire arabe-français (pp. 399-428) donne l'équivalent, ou les équivalents, français de tous les termes techniques selon leurs emplois dans le texte de Hुayyām.

2 L'œuvre mathématique de Hayyām, cet ouvrage en témoigne, n'est pas très volumineuse. Elle occupe portant une place privilégiée dans l'histoire des mathématiques, et cela pour des raisons expliquées dans les «commentaires mathématiques » ainsi que dans les deux «introductions » (pp. 3-29, pour les traités algébriques et pp. 271-9, pour le commentaire des difficultés des Eléments d'Euclide). Les commentaires de Hayyām touchent surtout deux points essentiels, débattus dès l'antiquité, des Eléments d'Euclide: la théorie des parallèles et la théorie des proportions. Quant à ses traités algébriques, Hayyām y présente, pour la première fois dans l'histoire des mathématiques, une théorie géométrique pour la résolution de toute équation dont le degré est égal à, ou moins de, trois. 
3 Le programme de Hayyām pour la résolution des équations, l'étude de Rashed (pp. 12-29) le montre, est, à plusieurs égards, celui repris, presque six siècles plus tard et sous un autre ciel, dans la Géométrie de Descartes. Cette étude souligne non seulement les points communs entre les deux programmes, mais aussi ceux où Descartes s'écarte de son prédécesseur pour ouvrir de nouvelles voies de recherches mathématiques. Ainsi, cette lecture moderne, et non modernisante, de l'œuvre de Hayyām la place dans l'histoire des mathématiques mondiales et souligne encore une fois l'actualité des travaux du savant iranien.

INDEX

Thèmes : 10. Histoire des Sciences et des Techniques

\section{AUTEURS}

HOSSEIN MASOUMI-HAMEDANI

Paris 